\title{
LncRNA HOTTIP Knockdown Attenuates Acute Myocardial Infarction via Regulating miR-92a-2/c-Met Axis
}

\author{
Beilei Wang ${ }^{1} \cdot$ Likun Ma $^{1} \cdot$ Junyi Wang ${ }^{2}$
}

Received: 29 July 2021 / Accepted: 21 December 2021 / Published online: 19 January 2022

(C) The Author(s) 2022

\begin{abstract}
Increasing investigations have focused on long non-coding RNAs (lncRNAs) in various human diseases, including acute myocardial infarction (AMI). Although lncRNA HOTTIP has been identified to play an important role in coronary artery diseases, its role and specific mechanism in AMI remain unclear. To investigate the potential role of HOTTIP in MI, HOTTIP expression in hypoxia-treated cardiomyocytes and myocardial tissues of MI mice was evaluated. The potential targets of HOTTIP and miR-92a-2 were predicted using Starbase and Targetscan. To further determine the cardio-protective effects of HOTTIP in vivo, si-HOTTIP and miR-92a-2 mimics were individually or co-injected into mice through intramyocardial injection. Moreover, their roles were further confirmed in rescue experiments. HOTTIP was significantly upregulated in ischemic myocardium of MI mice and hypoxia-induced cardiomyocytes. Moreover, HOTTIP knockdown markedly promoted cardiomyocyte growth and inhibited cardiomyocyte apoptosis in vitro. Luciferase reporter assay showed that HOTTIP could directly sponge miR-92a-2 to negatively regulate miR-92a-2 expression. In addition, c-Met was identified as a direct target of miR-92a-2, and their correlation was confirmed by luciferase reporter assay. MiR-92a-2 overexpression significantly enhanced the protective effect of HOTTIP knockdown against AMI through partially inhibiting c-Met expression. Our results demonstrated that HOTTIP downregulation attenuated AMI progression via the targeting miR-92a-2/c-Met axis and suggested that HOTTIP might be a potential therapeutic target for AMI.
\end{abstract}

Keywords AMI $\cdot$ HOTTIP $\cdot$ miR-92a-2 $\cdot$ c-Met $\cdot$ Apoptosis

\section{Introduction}

Cardiovascular disease has become the number one cause of death in the United States. Approximately over half of these deaths are due to acute myocardial infarction (AMI) [1,2]. Myocardial infarction (MI) is a leading cause of heart failure and a severe threat to human lives [3, 4]. It has been reported that cardiomyocyte apoptosis is closely associated with the

Handling Editor: Gen Suzuki

Likun Ma

1kma@ustc.edu.cn

1 Department of Cardiology, the First Affiliated Hospital of USTC, University of Science and Technology of China, Division of Life Sciences and Medicine, No.17 Lujiang Road, Hefei City 230036, Anhui Province, People's Republic of China

2 Institute of Ageing Research, Hangzhou Normal University School of Medicine, Hangzhou City 311121, Zhejiang Province, People's Republic of China pathogenesis of MI [5, 6] and is one of the mechanisms underlying AMI progression. Therefore, a better understanding of cardiomyocytes apoptosis is more urgent and may contribute to identifying potential therapeutic targets for AMI.

Long non-coding RNAs (lncRNAs), a newly identified class of non-protein-coding RNAs with more than 200 nucleotides in length, have been reported to participate in multiple heart diseases [7-9]. LncRNA HOTTIP is most abundantly expressed in various human cancers and plays an important role in cancer progression. For example, HOTTIP facilitates invasion, migration, and epithelialmesenchymal transition (EMT) of osteosarcoma cells by forming a positive feedback loop with c-Myc [10]. HOTTIP confers colorectal cancer cell metastasis and invasion by downregulating DKK1 [11]. HOTTIP promotes proliferation, invasion, and migration of papillary thyroid carcinoma cells via directly targeting miR-637 [12]. HOTTIP promotes endothelial cell proliferation and migration by activating the Wnt/ $\beta$-catenin signaling pathway [13]. Recently, HOTTIP 
has been identified to effectively alleviate oxygen-glucose deprivation-induced neuronal injury through regulating the miR-143/hexokinase 2 pathway [14]. However, the role of HOTTIP in AMI remains unclear.

C-Met, a receptor tyrosine kinase of hepatocyte growth factor (HGF), is expressed in various cells such as epithelial cells, hematopoietic cells, and neurons [15]. Many studies have revealed that aberrant activation of the $\mathrm{HGF} / \mathrm{c}-\mathrm{Met}$ signaling pathway is closely involved in cell proliferation, metastasis, and worse prognosis of patients with different gastrointestinal cancers [16], prostate cancer [17], nonsmall-cell lung cancer [18], colorectal cancer [19] and gastric cancer [20]. Sato et al. revealed that c-Met was significantly upregulated in the myocardium of MI patients, suggesting that c-Met directly affects the myocardium of MI patients [21]. In addition, c-Met/HGF signaling has beneficial effects against myocardial infarction and endothelial dysfunction through its pro-angiogenic, anti-inflammatory, and anti-fibrotic functions [22]. However, the regulatory network involving c-Met in AMI has not been well studied.

This study demonstrated that HOTTIP downregulation efficiently attenuated AMI progression through inhibiting cardiomyocyte apoptosis by directly targeting the miR-92a2/c-Met axis, suggesting that HOTTTIP might be a potential target for AMI treatment.

\section{Materials and Methods}

\section{Clinical Sample Acquisition}

Venous blood samples were collected from 42 MI patients (average age: $54.2 \pm 4.1$, male/female $=22 / 19$ ) and 42 healthy volunteers (average age: $54.6 \pm 3.2$, male/ female $=23 / 21$ ) in the First Affiliated Hospital of University of Science and Technology of China. After centrifugation, serum samples were collected and used for quantitative real-time polymerase chain reaction (qRT-PCR) to detect the expression patterns of lncRNA HOTTIP.

\section{Mouse Model of MI}

C57BL/6 mice (male, approximately 8-20 weeks) were obtained from Beijing Vital River Laboratory Animal Technology Co., Ltd. and kept under standard conditions. Mouse MI model was constructed through left anterior descending coronary artery (LAD) occlusion as previously described [23]. Mice were randomly divided into 5 groups, including sham group, MI group, si-HOTTIP group, miR-92a-2 group (miR-92a-2 mimics), and combined group (si-HOTTIP and miR-92a-2 mimics) with 7 mice in each group. $50 \mathrm{nM}$ of these si-RNAs or mimics were injected into left ventricles through intramyocardial injection each day for 1 week. Mice in all groups except the sham group were subjected to MI operation. At $12 \mathrm{~h}$ after MI, blood samples were collected for detection of serum myocardial enzyme activities. After that, mice were euthanized, and myocardial tissues were collected for the subsequent experiments. All procedures were approved by the Ethical Committee of the First Affiliated Hospital, Division of Life Sciences and Medicine, University of Science and Technology of China.

\section{Cell Isolation and Culture}

Mouse cardiomyocytes were isolated from 1 to 3-dayold neonatal C57BL/6 mice as previously described [24]. Briefly, the dissected hearts from mice were rinsed with HEPES-buffered saline solution, cut into pieces, and digested with $0.25 \%$ trypsin. Subsequently, cells were maintained in Dulbecco's modified Eagle medium/F-12 (DMEM/F-12; Gibco, Carlsbad, CA, USA) containing 5\% fetal bovine serum (Hyclone, Logan, UT, USA), $0.1 \mathrm{mM}$ ascorbate in insulin-transferring-sodium selenite media supplement (Sigma, St. Louis, MO), $100 \mathrm{U} / \mathrm{ml}$ penicillin, $100 \mu \mathrm{g} / \mathrm{ml}$ streptomycin, and $0.1 \mathrm{mM}$ bromodeoxyuridine (Gibco) in an atmosphere at $37{ }^{\circ} \mathrm{C}$ with $5 \% \mathrm{CO}_{2}$.

\section{Hypoxia/Re-oxygenation (H/R) Injury}

$\mathrm{H} / \mathrm{R}$ injury in cardiomyocytes was induced as previously described [25]. In brief, cardiomyocytes were cultured with serum-free medium under normoxic conditions for $24 \mathrm{~h}$. Cells were then cultured under hypoxia $\left(5 \% \mathrm{CO}_{2}, 95 \% \mathrm{~N}_{2}\right)$ for different times $(12,24$, and $48 \mathrm{~h})$ and subsequently under re-oxygenation condition $\left(5 \% \mathrm{CO}_{2}, 95 \% \mathrm{O}_{2}\right)$ for another $4 \mathrm{~h}$ to stimulate $\mathrm{H} / \mathrm{R}$ injury. In addition, control cells were cultured under normoxic conditions all the time. Finally, cells were harvested for the subsequent experiments.

\section{Cell Transfection}

Si-HOTTIP (siRNA against HOTTIP, 5'-AGGCTGAGC TAATACAGTA-3'), si-NC, miR-92a-2 mimics, miR-NC, and si-c-Met (siRNA against c-Met, 5'-ATCTTGAGCCAT TCACCGGAA-3') were purchased from Shanghai GenePharma Co., Ltd. (Shanghai, China) and transfected into mouse cardiomyocytes using Lipofectamine 2000 (Invitrogen). To overexpress HOTTIP and c-Met, the cDNA sequence of HOTTIP and c-Met were amplified with mouse genome as the template and cloned into pcDNA3.1 Expression vector to generate pc-HOTTIP and pc-c-Met. Cells transfected with the empty vector were used as the negative control (pc-NC). Similarly, pc-HOTTIP, pc-c-Met, or $\mathrm{pc}-\mathrm{NC}$ was transfected into mouse cardiomyocytes using Lipofectamine 2000. 


\section{CCK-8 assay}

Cell viability was evaluated using a Cell Counting Kit-8 (CCK-8, Dojindo Molecular Technologies, Gaithersburg, MD) as previously described [26]. Briefly, approximately $3 \times 10^{3}$ transfected cardiomyocytes were seeded into 96-well plates and incubated with additional CCK-8 reagent at 0,24 , 48,72 , and $96 \mathrm{~h}$ for another $4 \mathrm{~h}$. Finally, the absorbance at $450 \mathrm{~nm}$ was detected with a microplate reader.

\section{RNA Immunoprecipitation (RIP) Assay}

To determine the interaction between HOTTIP and miR92a-2, RIP assay was performed using the EZMagna RIP Kit (Millipore) following the manufacturer's instructions. Briefly, cells lysates were incubated with magnetic beads conjugated to Ago2 antibody or negative control IgG (Abcam) overnight at $4{ }^{\circ} \mathrm{C}$. On the next day, cell lysates were incubated with $30 \mu \mathrm{L}$ of magnetic beads for another $2 \mathrm{~h}$ at $4{ }^{\circ} \mathrm{C}$, and the immune-precipitated RNAs were purified and used for qRT-PCR analysis.

\section{RNA Extraction and qRT-PCR Analysis}

Total RNAs were extracted from myocardial tissues of MI mice or cultured cells using TRIzol Reagent (Invitrogen, Carlsbad, CA, USA). After reversely transcribing into cDNA using the PrimeScript RT reagent Kit (TaKaRa), qRT-PCR analysis was carried out using SYBR Green PCR Kit (Thermo) on an ABI 7500 Fast Real-Time PCR system (Applied Biosystems, Carlsbad, CA, USA). The expression of target genes was normalized to GAPDH or U6 using the $2^{-\Delta \Delta \mathrm{Ct}}$ method [27] with GAPDHA and U6 as the internal references. The primers used in this study were HOTTIP forward 5'-TCTGGTATTGCCTGGAACGCCAA-3' and reverse 5'-CAGTGGTAATCAAGTGGAGAATC-3', miR92a-2 forward 5'-GAGCTAGCGAATGGCACCCT-3' and reverse 5'-GCAGGAACGAAGTCGACTTA-3', c-Met forward 5'-GAGCCACTTAGAATCGAGGA-3' and reverse 5'-CTGAGGCTATAGATTCGTGCC-3', GAPDH forward 5'-CCAAGGTCATCCATGACAAC-3' and reverse 5'-GCT TCACCACCTTCTTGATG-3', and U6 forward 5'-AGA GAAGATTAGCATGGCCCCTG-3' and reverse 5'-AGT GCAGGGTCCGAGGTATT-3'.

\section{Western Blot}

Total proteins from cultured cells were extracted using RIPA Lysis Buffer (Beyotime, Shanghai, China). Approximately equal amounts of proteins were separated by $10 \%$ SDSPAGE and transferred onto PVDF membranes. After blocking with 5\% non-fat milk, the membranes were incubated with primary antibodies against c-Met (1:1000, Abcam),
Bax (1:1000, Abcam), Bcl-2 (1:1000, Abcam) and GAPDH (1:10,000, Cell Signaling Technology) overnight at $4{ }^{\circ} \mathrm{C}$. The membranes were subsequently exposed to horseradish peroxidase (HRP)-conjugated secondary antibodies for $2 \mathrm{~h}$ at room temperature. The protein bands of interest were detected using the ECL kit (Millipore), and protein levels were quantified using ImageJ software.

\section{Luciferase Reporter Assay}

Targetscan v7.2 and Starbase v.8 web tools were used to predict the binding site between HOTTIP and miR-92a-2 and between miR-92a-2 and c-Met. The wild type (WT) or mutant type (MUT) 3'-UTR of HOTTIP and c-Met containing the putative miR-92a-2 binding site was inserted into the pmirGLO vectors (Promega, Madison, WI, USA). Then mouse cardiomyocytes were co-transfected with luciferase reporter vectors and miR-92a-2 or miR-NC using Lipofectamine 2000 (Invitrogen). At $48 \mathrm{~h}$ post-transfection, relative luciferase activity was evaluated by the dual-luciferase reporter system.

\section{Apoptosis Assay}

Cardiomyocyte apoptosis was evaluated with Annexin V-FITC Apoptosis Kit (BD Biosciences). Briefly, cardiomyocytes under normoxia or hypoxia conditions were stained with Annexin V-fluorescein isothiocyanate (FITC) and propidium iodide (PI) and then analyzed by flow cytometry without re-oxygenation.

\section{Echocardiographic Evaluation}

The day before euthanization, mice in different groups were subjected to M-mode echocardiography to analyze left ventricular ejection fraction (LVEF), left ventricular fractional shortening (LVFS), left ventricular end-systolic diameter (LVESd) and left ventricular end-diastolic diameter (LVEDd) with ATL HDI 5000 ultrasound system with parameters of $15 \mathrm{MHz}$ linear and 12-MHz sectorial scan heads.

\section{Quantification of Infarct Size}

After euthanization, the myocardial tissues of mice were collected, cut into 5 - $\mu \mathrm{m}$-thick sections, embedded with paraffin, and stained with TTC solution for $15 \mathrm{~min}$. After fixation with formalin, samples were photographed using a digital camera, and infarct size was analyzed using Image $\mathbf{J}$ software (Olympus). 


\section{Immunohistochemistry (IHC) and Immunofluorescence (IF) Staining}

IHC and IF staining were performed as previously described [28]. Briefly, 5- $\mu$ m-thick paraffin-embedded sections of myocardial tissues were incubated with primary antibodies against c-Met (1:200, Abcam) and Cas-3 (1:200, Abcam) overnight at $4{ }^{\circ} \mathrm{C}$ respectively. After washed with PBS, the protein signals were visualized by incubating with Alexa FluorVR 647-labeled goat anti-rabbit IgG (ab150083, $1: 1500$, Abcam) and biotin-labeled goat anti-mouse $\operatorname{lgG}$ (ZSGB-Bio, Beijing, China) as secondary antibodies for $60 \mathrm{~min}$. The samples were covered using anti-fluorescence quenching tablets containing 4,6-diamidino-2-phenylindole (DAPI). The immunohistochemistry staining was stopped with a DAB chromogen kit (ZSGB-BIO, Beijing, China) before hematoxylin staining. The positive staining signals were observed under a fluorescence microscope (Nikon, Japan).

\section{TUNEL Assay and H\&E Staining}

Some $5 \mu$ m-thick paraffin-embedded sections of myocardial tissues were incubated with recombinant terminal deoxynucleotidyl transferase (rTdT) solution at $37{ }^{\circ} \mathrm{C}$ for $1 \mathrm{~h}$ in the dark and stained with DAPI solution. TUNEL stained cells were observed under a fluorescence microscope. The apoptotic index was evaluated as the percentage of TUNELpositive cells to the total cells. In addition, some sections were stained with hematoxylin-eosin solution and observed under an optical microscope as previously described [29].

\section{Detection of CK-MB and LDH in Serum}

Serum creatine kinase MB (CK-MB) and lactate dehydrogenase $(\mathrm{LDH})$ levels in mice from different groups were detected using a Creatine Kinase Activity Assay kit and a LDH Colorimetric Assay kit, respectively.

\section{Statistical Analysis}

Data were presented as mean \pm SD derived from at least three independent experiments and statistically analyzed using GraphPad Prism software. Differences were determined by Student's $t$ test (two groups) or one-way ANOVA (among three or more groups). $P<0.05$ was considered statistically significant.

\section{Results}

\section{LncRNA HOTTIP is Significantly Upregulated in the Clinical Serum Samples, Myocardial Tissues of MI Mice, and Hypoxia-Treated Cardiomyocytes}

HOTTIP expression in the serum samples of MI patients was detected. The results showed that HOTTIP expression was upregulated in MI patients compared with the healthy controls (Fig. s1). To investigate the potential role of HOTTIP in MI, HOTTIP expression in hypoxia-treated cardiomyocytes and myocardial tissues of MI mice was evaluated. The results indicated that HOTTIP was significantly upregulated in hypoxia-treated cardiomyocytes in a time-dependent manner $(p<0.05)$ (Fig. 1A) and in myocardial tissues of MI mice compared with the sham mice $(p<0.01)$ (Fig. 1B). In addition, miR-92a-2 was downregulated in mouse cardiomyocytes exposed to hypoxia in a time-dependent manner $(p<0.05)$ (Fig. 1C). Consistent with the findings in vitro, miR-92a-2 was also obviously downregulated in myocardial tissues of MI mice compared with the sham mice $(p<0.01)$ (Fig. 1D). These results suggested that HOTTIP and miR92a-2 might play crucial roles in ischemic cardiac injury.

\section{HOTTIP Serves as a Sponge of miR-92a-2}

The potential targets of HOTTIP were predicted using Starbase. The results revealed a putative binding site between HOTTIP and miR-92a-2 (Fig. 2A), suggesting that miR92a-2 might be a target of HOTTIP. Luciferase reporter assay was performed and indicated that miR-92a-2 mimics significantly decreased the relative luciferase activity of WT HOTTIP vector $(p<0.01)$, while exhibited no impact on the MUT vector (Fig. 2B). Meanwhile, RIP assay using antiAgo2 was carried out and found that both HOTTIP and miR92a-2 were significantly enriched in immune-precipitates containing anti-Ago 2 compared with that containing negative control anti-IgG $(p<0.01)$ (Fig. 2C). In addition, mouse cardiomyocytes were transfected with si-HOTTIP, si-NC, pc-HOTTIP or pc-NC, and the expression of HOTTIP and miR-92a-2 was evaluated by qRT-PCR. The results showed that si-HOTTIP significantly decreased HOTTIP expression $(p<0.01)$ and increased miR-92a-2 expression compared with si-NC $(p<0.001)$, while pc-HOTTIP markedly increased HOTTIP expression $(p<0.001)$ and decreased miR-92a-2 expression compared with pc-NC $(p<0.01)$ (Fig. 2D). Furthermore, the expression level of MiR-1792a-1 Cluster Host Gene (MIR17HG) did not decrease in the myocardial tissues of MI mice (Fig. s2). These results 
A

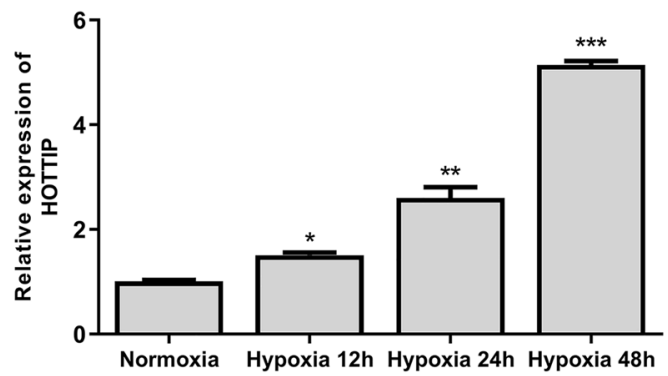

C

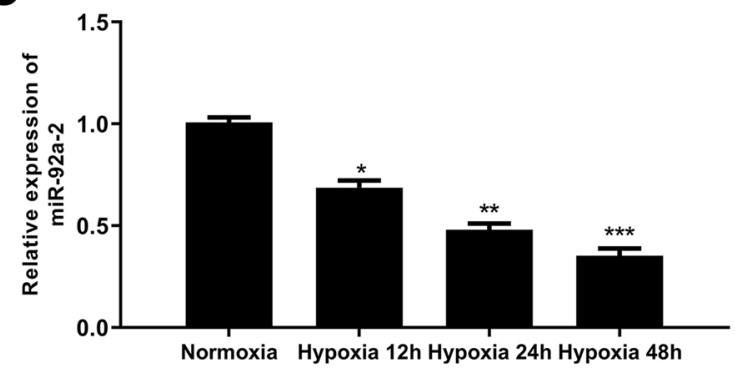

Fig. 1 Ischemic cardiac injury increased HOTTIP expression and decreased miR-92a-2 expression. A and C HOTTIP A and miR92a-2 C mRNA levels in hypoxia-treated mouse cardiomyocytes were
B

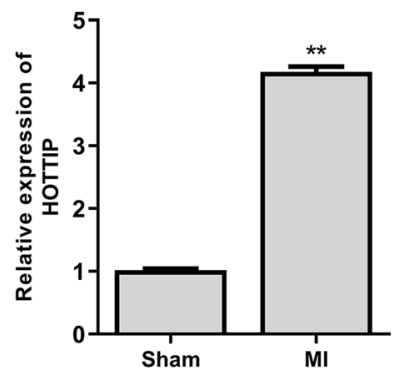

D

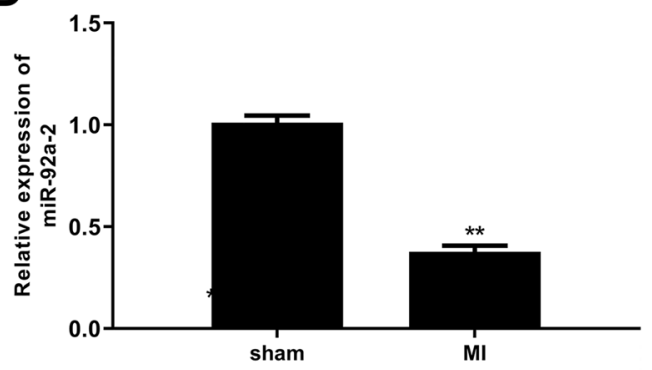

evaluated by qRT-PCR $(n=3)$. B and D HOTTIP $\mathbf{B}$ and miR-92a-2 D mRNA levels in myocardial tissues of MI mice were evaluated by qRT-PCR $(n=6) .{ }^{*} P<0.05,{ }^{* *} P<0.01,{ }^{* * *} P<0.001$
Fig. 2 LncRNA HOTTIP serves as a sponge of miR-92a- 2 . A

The putative interaction region between HOTTIP and miR92a-2 was predicted by Starbase. B WT and MUT 3'UTR of HOTTIP were co-transfected with miR-92a-2 mimics or miR-NC into cardiomyocytes, and relative luciferase activity was evaluated by dual-luciferase reporter system. $\mathbf{C}$ The relative enrichment of HOTTIP and miR-92a-2 was determined using anti-Ago2 RIP assay, with anti-IgG as the negative control. D Cardiomyocytes were transfected with si-HOTTIP, si-NC, pc-HOTTIP, or pc-NC, and the mRNA expression of HOTTIP and miR-92a-2 was evaluated by qRT-PCR. $n=3,{ }^{* *} P<0.01$, ${ }^{* * *} P<0.001$
A

miR-92a-2-5p GGGUGGGGAUUUGUUGCAUUAC $:::::::$ :

HOTTIP
B

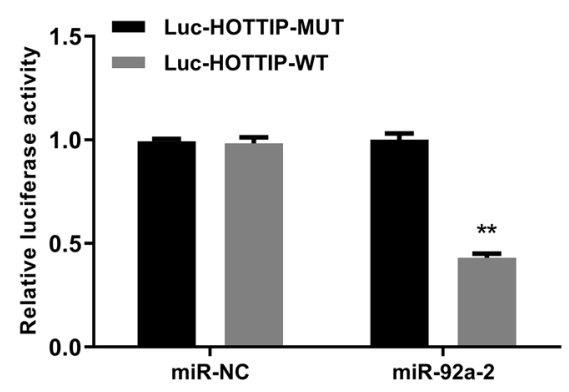

C

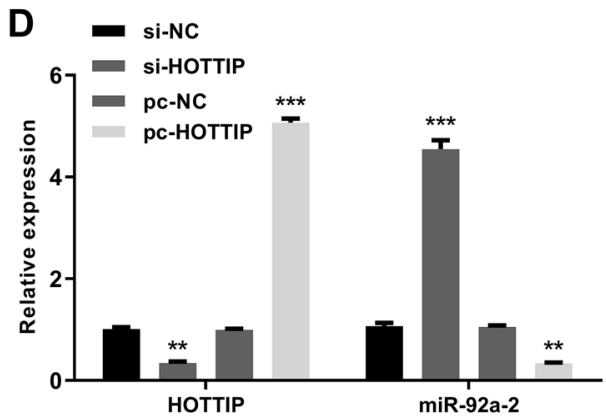


A

miR-92a-2-5p GGGUGGGGAUUUGUUGCAUUAC

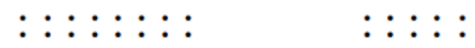

c-Met
B
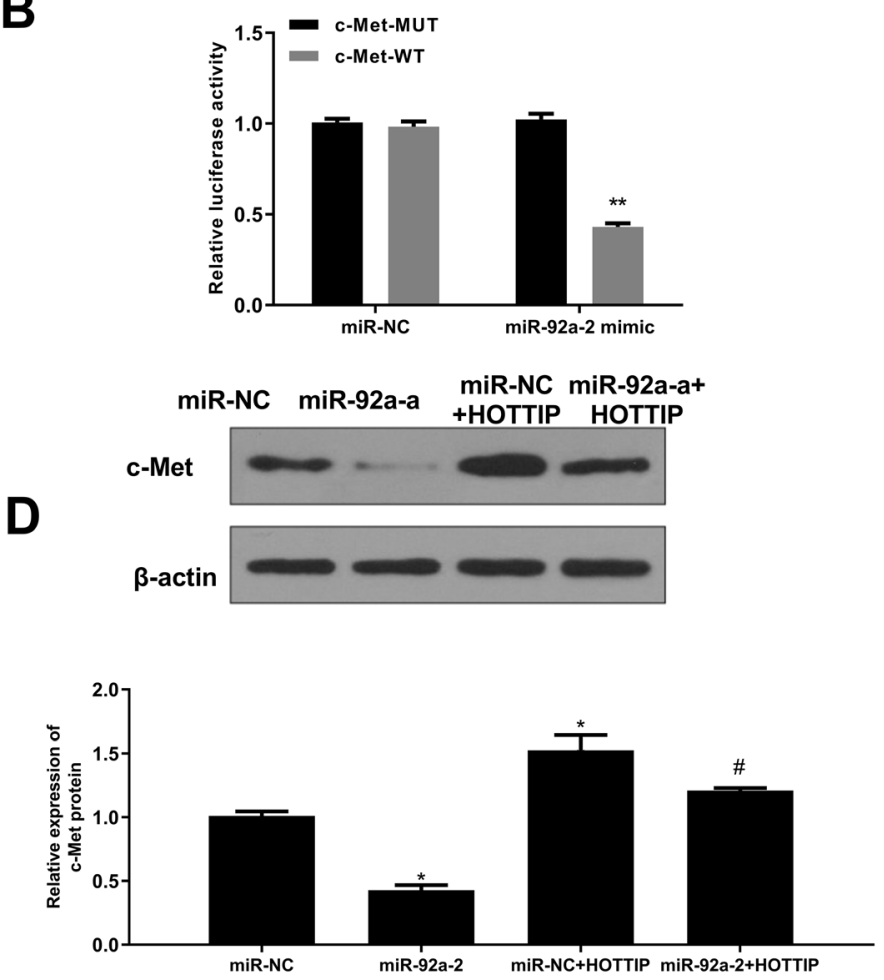

$\mathbf{F}$

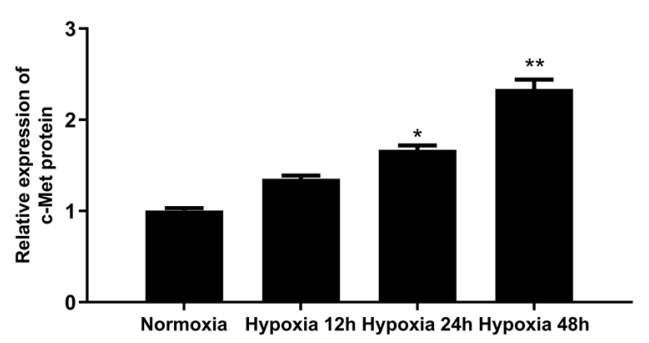

level was evaluated by Western blot $(n=3)$. $\mathbf{E}$ c-Met expression in myocardial tissues of MI and sham mice was observed by IHC assay $(n=6)$. F c-Met mRNA level in hypoxia-treated mouse cardiomyocytes was evaluated by qRT-PCR $(n=3) .{ }^{*} P<0.05,{ }^{* *} P<0.01$, ${ }^{* * *}$ $P<0.001$ vs. miR-NC or normoxia condition. ${ }^{\#} P<0.05,{ }^{\#} P<0.01$ vs. miR-92a-2 mimics group

of MUT c-Met vector (Fig. 3B). Mouse cardiomyocytes were transfected with miR-92a-2 mimics, miR-NC, or cotransfected with miR-NC and pc-HOTTIP or miR-92a-2 mimics and pc-HOTTIP. QRT-PCR indicated that miR92a-2 mimics significantly increased miR-92a-2 expression compared with miR-NC $(p<0.001)$, and pc-HOTTIP decreased miR-92a-2 expression compared with miR-NC $(p<0.01)$, while co-transfection of miR-92a-2 mimics and pc-HOTTIP obviously reversed the effect of miR-92a-2 mimics $(p<0.01)$ (Fig. 3C). Meanwhile, miR-92a-2 mimics markedly decreased c-Met expression at both mRNA with miR-NC $(p<0.01)$ but had no obvious effect on that 
$(p<0.01)$ (Fig. 3C) and protein $(p<0.01)$ (Fig. 3D) levels compared with miR-NC. HOTTIP overexpression significantly increased c-Met expression at both mRNA $(p<0.01)$ (Fig. 3C) and protein $(p<0.01)$ (Fig. 3D) levels compared with miR-NC. By contrast, co-transfection of miR-92a-2 mimics and pc-HOTTIP obviously reversed the effect of miR-92a-2 mimics on c-Met expression at mRNA level $(p<0.01)$ (Fig. 3C) and protein level $(p<0.05)$ (Fig. 3D). In addition, IHC assay showed that c-Met expression was obviously increased in myocardial tissues of MI mice compared with that of the sham mice (Fig. 3E). Similarly, c-Met expression was increased in hypoxia-treated cardiomyocytes in a time-dependent manner $(p<0.05)$ (Fig. 3F). These results suggested that HOTTIP might elevate c-Met expression through sponging miR-92a-2 in MI.

\section{HOTTIP Knockdown Promotes Growth and Inhibits Apoptosis of Hypoxia-Treated Cardiomyocytes In Vitro}

Next, cardiomyocytes were transfected with si-HOTTIP, si-NC, miR-92a-2 mimics, or co-transfected with miR92a-2 mimics and si-HOTTIP, and cell viability and apoptosis were evaluated. As shown in Fig. 4A and Fig. 4B, hypoxia significantly decreased viability and induced apoptosis of cardiomyocytes compared with normoxia condition $(p<0.001)$, and si-HOTTIP and miR-92a-2 mimics significantly promoted viability and inhibited apoptosis of hypoxiatreated cardiomyocytes compared with si-NC $(p<0.01)$, and co-transfection of miR-92a-2 mimics and si-HOTTIP further enhanced cell viability and inhibited apoptosis of
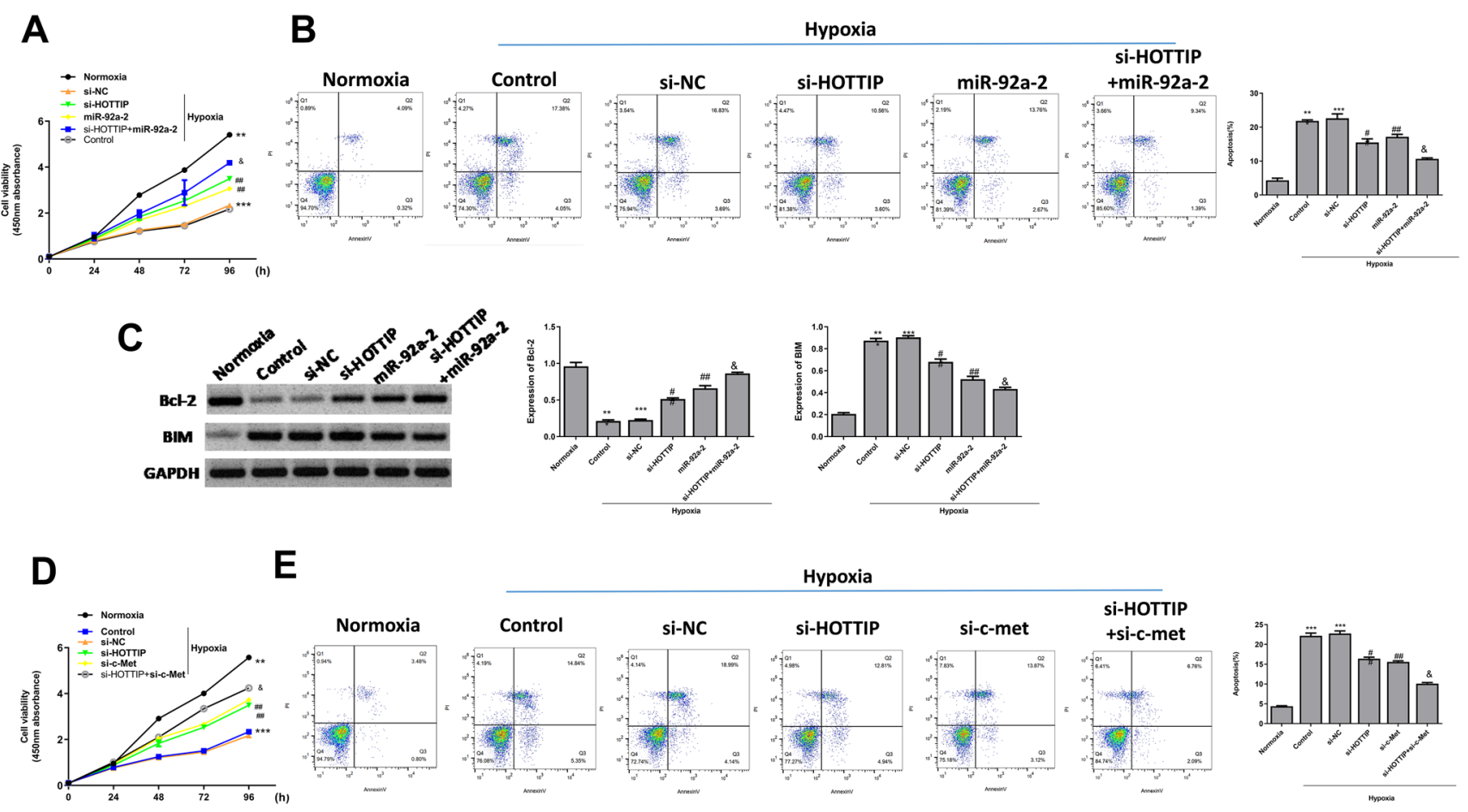

$\mathbf{E}$

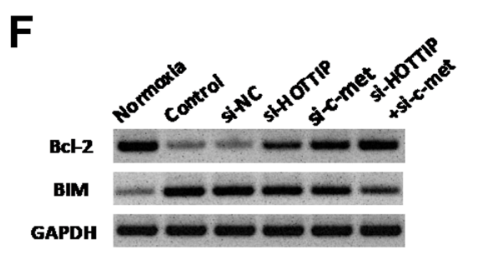

Fig. 4 HOTTIP knockdown promotes growth and inhibits apoptosis of hypoxia-treated cardiomyocytes in vitro. A and $\mathbf{B}$ Mouse cardiomyocytes were transfected with si-HOTTIP, si-NC, miR-92a-2 mimics, or co-transfected with miR-92a-2 mimics and si-HOTTIP and then treated with hypoxia or normoxia conditions. A Cell viability was evaluated by CCK- 8 assay. B Cell apoptosis was evaluated by flow cytometry assay. C Expressions of bcl-2 and BIM were evaluated by Western blot. D and E Mouse cardiomyocytes were trans- fected with si-HOTTIP, si-NC, si-c-Met, or co-transfected with siHOTTIP and si-c-Met, and then treated with hypoxia or normoxia conditions. D Cell viability was evaluated by CCK-8 assay. E Cell apoptosis was evaluated by flow cytometry assay. F Expressions of bcl-2 and BIM were evaluated by Western blot. $n=3,{ }^{* *} P<0.01$, ${ }^{* * * *} P<0.001$ vs. normoxia condition; ${ }^{\# \#} P<0.01 \mathrm{vs}$. si-NC group; \& $P<0.05$ vs. si-HOTTIP group 
hypoxia-treated cardiomyocytes compared with si-HOTTIP group $(p<0.05)$. We further detected the expression of Bcl-2 and BIM. The results showed that hypoxia significantly decreased bcl-2 expression and induced BIM expression compared with normoxia condition $(p<0.001)$, and si-HOTTIP and miR-92a-2 mimics significantly promoted bcl-2 expression and inhibited BIM expression compared with si-NC in hypoxia-treated cardiomyocytes $(p<0.01)$. Furthermore, co-transfection of miR-92a-2 mimics and siHOTTIP further enhanced the effect of si-HOTTIP (Fig. 4C, $p<0.05$ ). Similarly, the results of CCK-8 assay (Fig. 4D) and flow cytometry assay (Fig. 4E) showed that both si-cMet and si-HOTTIP significantly promoted viability and inhibited apoptosis of hypoxia-treated cardiomyocytes compared with si-NC $(p<0.01)$, and co-transfection of si-c-Met and si-HOTTIP further enhanced cell viability and inhibited apoptosis of hypoxia-treated cardiomyocytes compared with si-HOTTIP group $(p<0.05)$. We also found that both si-cMet and si-HOTTIP significantly promoted bcl-2 expression and inhibited BIM expression in hypoxia-treated cardiomyocytes compared with si-NC (Fig. 4F, $p<0.01$ ). We further demonstrated that c-Met overexpression reversed the protective effect of si-HOTTIP or miR-92a-2 mimics on AMI progression, including cardiomyocyte apoptosis (Fig. s3). These results indicated that HOTTIP knockdown promoted growth and inhibited apoptosis of hypoxia-treated cardiomyocytes by modulating the miR-92a-2/c-Met axis in vitro.

\section{HOTTIP Knockdown Alleviates Myocardial Function Through Upregulating miR-92a-2 In Vivo}

To further determine the cardio-protective effects of HOTTIP in vivo, si-HOTTIP, miR-92a-2 mimics, or si-HOTTIP plus miR-92a-2 mimics were injected into mice through intramyocardial injection. The results of qRT-PCR showed that si-HOTTOP significantly decreased HOTTIP expression $(p<0.01)$, increased miR-92a-2 expression $(p<0.01)$, and decreased c-Met expression $(p<0.01)$; MiR-92a-2 mimics markedly increased miR-92a-2 expression $(p<0.01)$ and decreased c-Met expression $(p<0.01)$; Co-injection of siHOTTIP and miR-92a-2 mimics obviously further elevated the effect of si-HOTTIP on the expression of miR-92a-2 $(p<0.05)$ and c-Met $(p<0.05)$ (Fig. 5A). Meanwhile, siHOTTIP and miR-92a-2 mimics all significantly decreased c-Met protein level $(p<0.05)$, and si-HOTTIP plus miR92a-2 mimics further decreased c-Met protein level compared with si-HOTTIP group $(p<0.05)$ (Fig. 5B). In addition, we found that the infarct size of myocardial tissues was significantly increased in the MI group and HOTTIP knockdown and miR-92a-2 mimics obviously decreased infarct size. Meanwhile, the inhibitory effect of si-HOTTIP was significantly enhanced by miR-92a-2 mimics (Fig. 5C). In addition, cardiac function indexes including LVEF (Fig. 5D),
LVFS (Fig. 5E), LVESd (Fig. 5F), and LVEDd (Fig. 5G) of different groups were evaluated by echocardiographic measurement. The results indicated that LVEF and LVFS of myocardial tissues in MI group were significantly decreased, while LVESd and LVEDd of myocardial tissues in MI group were increased compared with the sham group $(p<0.01)$. HOTTIP knockdown led to a significant increase in LVEF and LVFS and a marked decrease in LVESd and LVEDd $(p<0.05)$. Meanwhile, the protective effect of si-HOTTIP was significantly enhanced by miR-92a- 2 overexpression $(p<0.05)$. These results revealed that HOTTIP downregulation could effectively alleviate myocardial dysfunction through upregulating miR-92a-2 in vivo.

\section{HOTTIP Knockdown Improves Myocardial Injury and Apoptosis via Sponging miR-92a-2 In Vivo}

We also evaluated the degree of myocardial cell injury by measuring serum CK-MB and LDH levels. The results (Fig. 6A and B) showed that serum CK-MB and LDH levels were significantly increased in the MI group than in the sham group $(p<0.001)$, and both si-HOTTIP and miR-92a-2 mimics markedly decreased serum CK-MB and LDH levels $(p<0.01)$. Meanwhile, miR-92a-2 mimics significantly enhanced the inhibitory effect of si-HOTTIP on serum CK-MB and LDH levels $(p<0.05)$. The myocardial infarct size was increased in all groups except for the sham group $(p<0.05)$. Si-HOTTIP and miR-92a-2 mimics significantly decreased the myocardial infarct size $(p<0.05)$, while the inhibitory effect of si-HOTTIP was enhanced by miR-92a-2 mimics $(p<0.05)$ (Fig. 6C). TUNEL staining assay was used to evaluate cardiomyocyte apoptosis rate in mouse heart tissues, and the results indicated that apoptosis rate was significantly increased in MI group than in the sham group $(p<0.05)$. Both si-HOTTIP and miR-92a-2 mimics markedly decreased apoptosis in MI mice $(p<0.05)$. Meanwhile, the inhibitory effect of si-HOTTIP was obviously enhanced by miR-92a-2 mimics $(p<0.05)$ (Fig. 6D and E). H\&E staining assay (Fig. 6E) also showed a similar trend with TUNEL staining assay. In other words, cardiomyocyte apoptosis was significantly increased in MI group than in the sham group, and both si-HOTTIP and miR-92a-2 mimics obviously inhibited cardiomyocyte apoptosis. Furthermore, the protective effect of si-HOTTIP was obviously enhanced by miR-92a-2 mimics. IF staining also showed that Cas-3 expression in MI was inhibited when treated with si-HOTTIP or miR-92a-2 mimics (Fig. s4). In addition, the apoptosis-related makers, including pro-apoptotic factor Bax and anti-apoptotic factor Bcl-2, in mouse heart tissues was evaluated by Western blot (Fig. 6F). The results indicated that Bax was significantly upregulated $(p<0.001)$, while $\mathrm{Bcl}-2(p<0.001)$ was downregulated in MI mice compared with the sham mice. Si-HOTTIP and miR-92a-2 mimics 
A

B

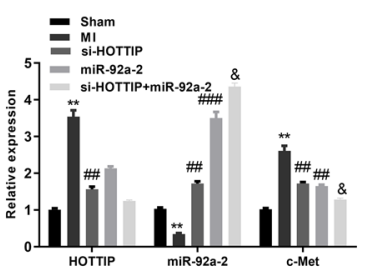

C
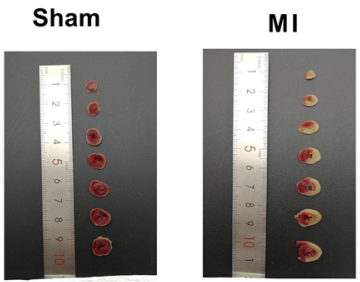

D

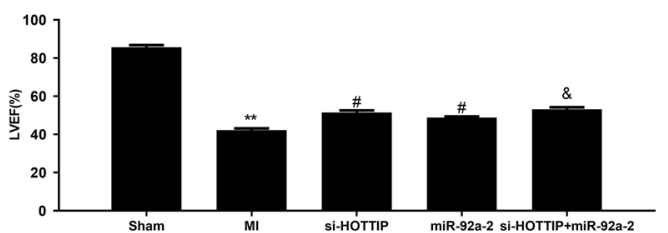

$\mathbf{F}$

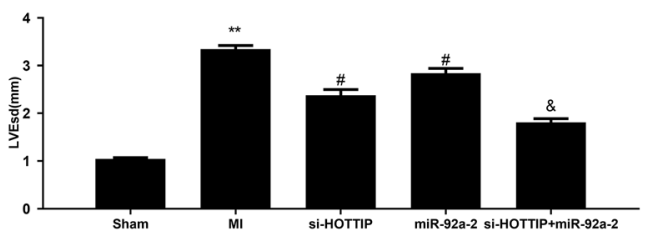

Fig. 5 HOTTIP knockdown alleviates myocardial function via upregulating miR-92a-2 in vivo. A The mRNA levels of HOTTIP, miR92a-2 and c-Met in myocardial tissues of mice from different groups were evaluated by qRT-PCR. B c-Met protein levels in myocardial tissues of mice from different groups were evaluated by Western blot.

markedly decreased Bax expression $(p<0.05)$ and increased Bcl-2 expression $(p<0.05)$, and si-HOTTIP plus miR-92a-2 mimics further enhanced $\mathrm{Bcl}-2$ expression $(p<0.05)$ and decreased Bax expression $(p<0.05)$. These results demonstrated that HOTTIP downregulation effectively improved myocardial injury and inhibited cardiomyocyte apoptosis by targeting miR-92a-2 in vivo.

\section{Discussion}

Increasing studies have suggested that lncRNAs play important roles in gene expression involved in the development of AMI [30]. IncRNA MIAT regulates microvascular
si-HOTTIP+
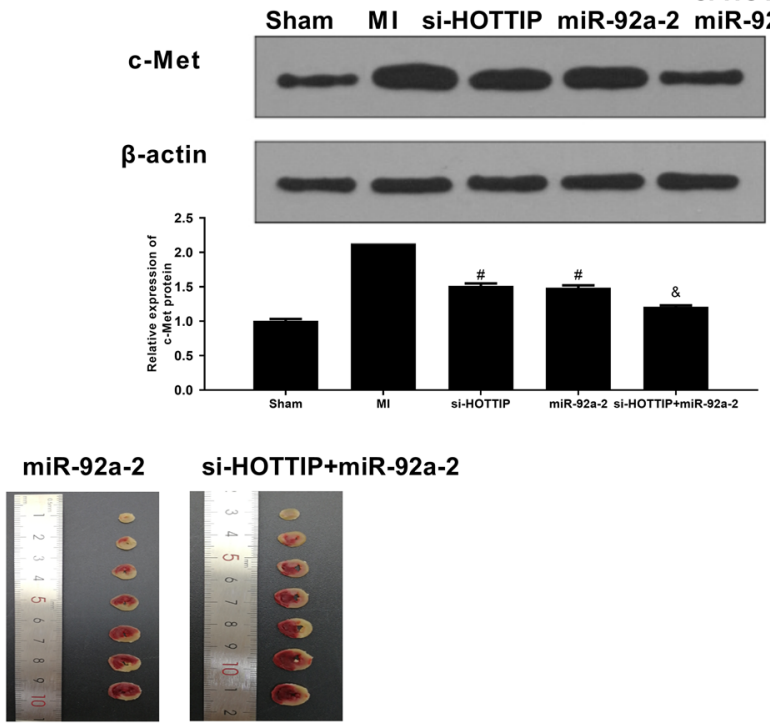

$\mathbf{E}$

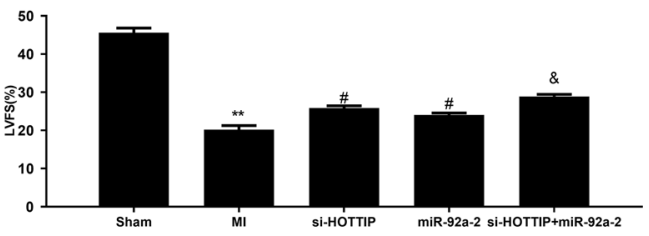

G

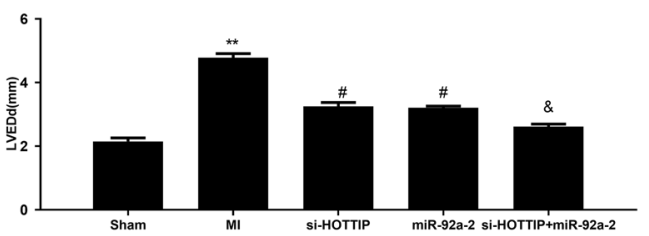

C The infarct sizes of mice from different groups were evaluated by TTC staining assay. D-G Cardiac injury indexes including LVEF D, LVFS E, LVESd $\mathbf{F}$, and LVEDd $\mathbf{G}$ of different groups were evaluated by echocardiographic measurement. ${ }^{* *} P<0.01 \mathrm{vs}$. sham group; ${ }^{\#}$ $P<0.05$ vs. MI group; ${ }^{\&} P<0.05$ vs. si-HOTTIP group

dysfunction by acting as a competing endogenous RNA (ceRNA) of miR-150-5p [31]. LncRNA GAS5 regulates apoptosis of macrophages and vascular endothelial cells in atherosclerosis[32]. Since AMI is the first manifestation of ischemic heart disease, it has drawn more and more attention [33]. Over the past decades, studies have revealed that AMI progression, especially cardiomyocyte proliferation and apoptosis, is closely related to many lncRNAs, some of which might be potential biomarkers for AMI [34]. For instance, ANRIL knockdown relieves myocardial cell apoptosis during AMI by regulating the expression of IL-33/ST2 [35]. Inhibition of lncRNA Mirt1 significantly attenuates AMI via suppressing cardiomyocyte apoptosis and reduces inflammatory cell infiltration by inhibiting the activation of 
A

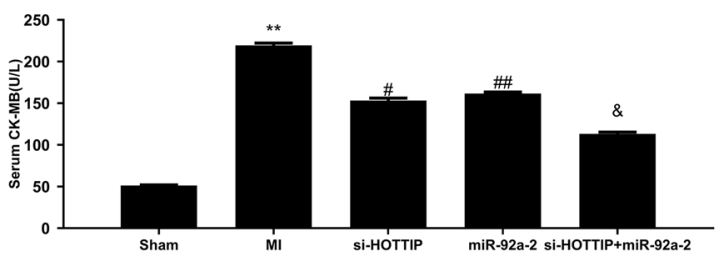

C

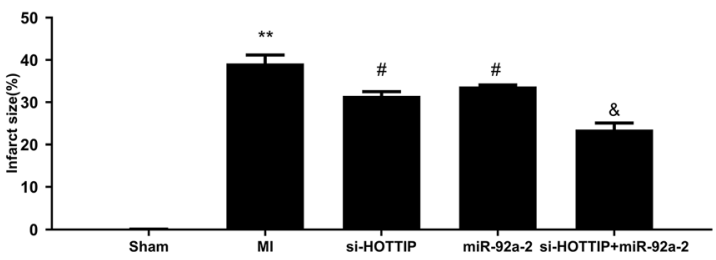

E
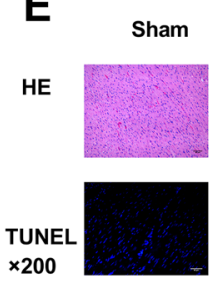

$\times 400$

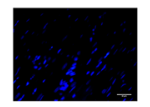

\section{MI}
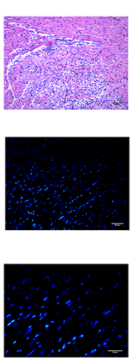

si-HOTTIP
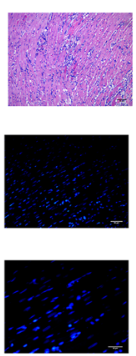

B

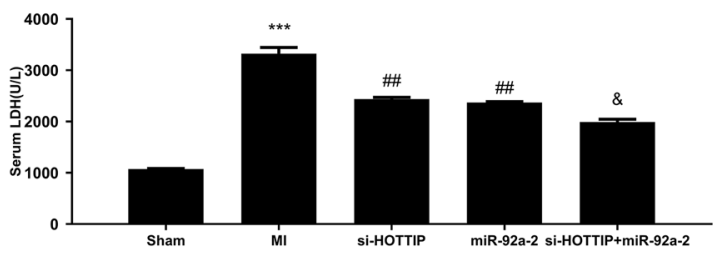

D

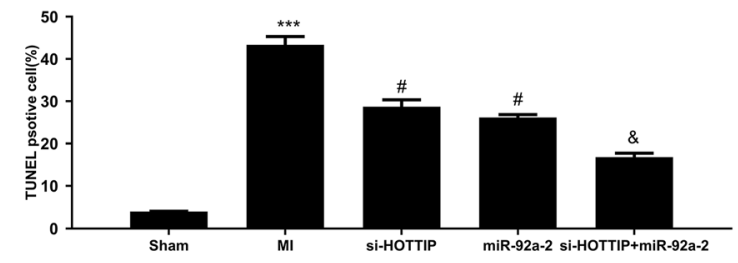

$\mathbf{F}$

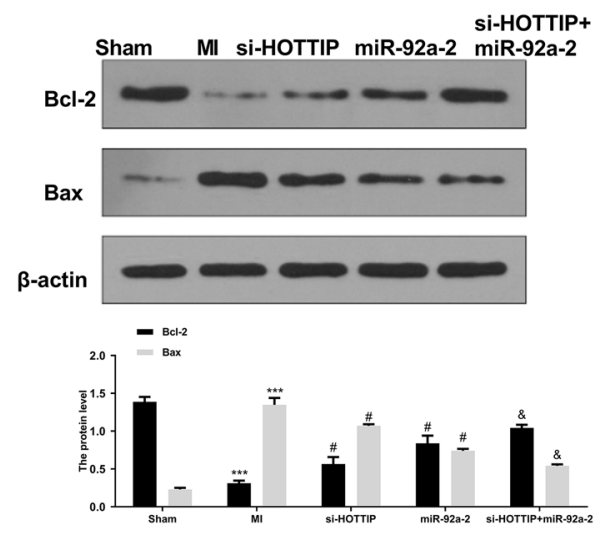

Fig. 6 HOTTIP knockdown improves myocardial injury via sponging miR-92a-2. A and B The serum CK-MB A and LDH B levels were evaluated by respective detection kits. C Myocardial infarct size. D Apoptosis rate of cardiomyocytes was quantified from TUNEL staining assay. E Cardiomyocyte apoptosis was observed by TUNEL

NF-kB signaling pathway [36]. LncRNA Gm2691 overexpression attenuates cardiomyocyte apoptosis and inflammatory responses after MI via modulating PI3K/Akt signaling pathway [37]. LncRNA TUG1 downregulation ameliorates myocardial injury during ischemia and reperfusion by regulating HMGB 1- and Rac1-mediated autophagy via directly upregulating miR-142-3p [38]. LncRNA Kcnq1ot1 renders cardiomyocyte apoptosis during AMI via upregulating Tead 1 expression by directly sponging miR- $466 \mathrm{k}$ and $\mathrm{miR}$ 466i-5p [39]. LncRNA XIST upregulation effectively inhibits hypoxia-induced cardiomyocyte apoptosis by targeting miR-150-5p/Bax axis during AMI progression [40]. In addition, IncRNAs SOX2-OT [41], RMRP [42], HULC [43] and MHRT [44] are also involved in regulating hypoxia-treated cardiomyocyte apoptosis. Although lncRNA HOTTIP has been identified to play crucial roles in coronary artery diseases, its effect and specific mechanism in AMI have not and $\mathrm{H} \& \mathrm{E}$ staining assay. $\mathbf{F}$ Bax and Bcl-2 protein levels in mouse heart tissues were evaluated by Western blot. $n=6,{ }^{* *} P<0.01$, ${ }^{* * *}$ $P<0.001$ vs. sham group; ${ }^{\#} P<0.05,{ }^{\#} P<0.01$ vs. MI group; \& $P<0.05$ vs. si-HOTTIP group

been reported. In this study, we, for the first time, revealed that HOTTIP is significantly upregulated in myocardial tissues of MI mice and hypoxia-treated cardiomyocytes, suggesting a potential role of HOTTIP in AMI.

Recent reports have also revealed that many miRNAs are involved in cardiomyocyte apoptosis during AMI. For example, miR-124 regulates oxidative stress- and hypoxiainduced cardiomyocyte apoptosis by targeting Dhcr24, suggesting that miR-124/Dhcr24 axis might be a potential biomarker for AMI [45]. MiR-155 promotes AMI-induced cardiomyocyte apoptosis through targeting QKI [46]. MiR214 is highly expressed in the sera of elderly AMI patients, and its upregulation inhibits myocardial cell apoptosis by decreasing the expression of miR-214 target genes, including PUMA, PTEN, Bax, and caspase 7 [47]. In this study, we predicted the potential targets of IncRNA HOTTIP using Starbase v2.0 and showed that miR-92a-2 is a target 
of HOTTIP. Luciferase reporter assay and RIP assay also confirmed that HOTTIP could directly sponge miR-92a-2. Moreover, we found that the expression level of miR-1792a-1 cluster host gene (MIR17HG) is not changed in the myocardial tissues of MI mice, indicating that HOTTIP specifically decreases miR-92a-2 level and miR-92a-2 level is modulated during AMI process in mice. Moreover, cotransfection of miR-92a-2 mimics and si-HOTTIP further enhances viability and inhibits apoptosis of hypoxia-treated cardiomyocytes than transfection with si-HOTTIP alone both in vitro and in vivo, suggesting the important roles of miR-92a-2 in AMI.

C-Met has been identified as a direct target of miRNAs to participate in important biological processes. Cheng et al. revealed that miR-449a inhibits hepatocellular carcinoma cell growth via G1 phase arrest and activates HGF/MET c-Met signaling pathway [48]. C-Met is involved in miR433-mediated inhibition of the EMT process in bladder cancer through modulating the Akt/GSK-3 $\beta /$ Snail signaling pathway [49]. In this study, we identified c-Met as a target of miR-92a-2 and showed that miR-92a-2 mimics significantly decreases the relative luciferase activity of WT c-Met vector but not MUT c-Met vector compared with miR-NC. In addition, c-Met the expression is markedly decreased by miR92a-2 mimics but increased by HOTTIP overexpression, and co-transfection of miR-92a-2 mimics and pc-HOTTIP obviously reverses the effect of miR-92a-2 mimics on c-Met expression. Moreover, miR-92a-2 mimics plus si-HOTTIP significantly enhance the protective effect of si-HOTTIP on myocardial function in vivo and cardiomyocytes apoptosis in vitro. Bcl-2 family comprises both cell death inhibiting and promoting proteins, such as anti-apoptotic factor Bcl-2 and pro-apoptotic factor Bax [50]. We found that si-HOTTIP and miR-92a-2 mimics markedly decrease Bax expression and increase Bcl-2 expression in mouse heart tissues, and si-HOTTIP plus miR-92a-2 mimics further enhance Bcl-2 expression and decrease Bax expression. These results demonstrated that the protective effects of si-HOTTIP and miR92a-2 mimics in AMI are mediated by cardiomyocytes apoptosis. However, whether c-Met overexpression could reverse the protective effect of si-HOTTIP or miR-92a-2 mimics in AMI progression, including cardiomyocytes apoptosis, should be determined in the subsequent experiments. In addition, our results are obtained in mouse-derived samples and need to be further clarified in myocardial tissues of AMI patients.

\section{Conclusion}

Our study revealed a new regulatory mechanism of lncRNA HOTTIP in AMI: IncRNA HOTTIP downregulation could effectively attenuate AMI progression via targeting the
miR-92a-2/c-Met axis, suggesting that HOTTIP might be a potential therapeutic target for AMI.

Supplementary Information The online version contains supplementary material available at https://doi.org/10.1007/s12012-021-09717-3.

Authors' Contributions BLW: manuscript writing, literature research, data analysis and statistical analysis; JYW: literature search, clinical research and data analysis; LKM: study design, funding acquisition and manuscript review. All authors read and approved the final manuscript.

Funding No funding was received.

Data Availability The datasets used and/or analyzed during the current study are available from the corresponding author on reasonable request.

\section{Declarations}

Conflict of interest The authors declare that they have no conflict of interest.

Research Involving Animals All procedures were approved by the Ethical Committee of the First Affiliated Hospital of USTC, Division of Life Sciences and Medicine, University of Science and Technology of China.

Informed Consent Not applicable.

Consent for Publication Not applicable.

Open Access This article is licensed under a Creative Commons Attribution 4.0 International License, which permits use, sharing, adaptation, distribution and reproduction in any medium or format, as long as you give appropriate credit to the original author(s) and the source, provide a link to the Creative Commons licence, and indicate if changes were made. The images or other third party material in this article are included in the article's Creative Commons licence, unless indicated otherwise in a credit line to the material. If material is not included in the article's Creative Commons licence and your intended use is not permitted by statutory regulation or exceeds the permitted use, you will need to obtain permission directly from the copyright holder. To view a copy of this licence, visit http://creativecommons.org/licenses/by/4.0/.

\section{References}

1. Reed, G. W., Rossi, J. E., \& Cannon, C. P. (2017). Acute myocardial infarction. Lancet (London, England), 389(10065), 197-210. https://doi.org/10.1016/s0140-6736(16)30677-8

2. Pollard, T. J. (2000). The acute myocardial infarction. Primary Care, 27(3), 631-649. https://doi.org/10.1016/s0095-4543(05) 70167-6

3. Castro-Dominguez, Y., Dharmarajan, K., \& McNamara, R. L. (2018). Predicting death after acute myocardial infarction. Trends in Cardiovascular Medicine, 28(2), 102-109. https://doi.org/10. 1016/j.tcm.2017.07.011

4. Mehta, L. S., Beckie, T. M., DeVon, H. A., Grines, C. L., Krumholz, H. M., Johnson, M. N., Lindley, K. J., Vaccarino, V., Wang, T. Y., Watson, K. E., \& Wenger, N. K. (2016). Acute myocardial infarction in women: A scientific statement from the American 
Heart Association. Circulation, 133(9), 916-947. https://doi.org/ 10.1161/cir.0000000000000351

5. Burgos, J. I., Morell, M., Mariángelo, J. I. E., \& Vila Petroff, M. (2019). Hyperosmotic stress promotes endoplasmic reticulum stress-dependent apoptosis in adult rat cardiac myocytes. Apoptosis : an International Journal on Programmed Cell Death, 24(9-10), 785-797. https://doi.org/10.1007/s10495-019-01558-4

6. Zhou, Y., Richards, A. M., \& Wang, P. (2019). MicroRNA-221 Is Cardioprotective and Anti-fibrotic in a Rat Model of Myocardial Infarction. Molecular Therapy-Nucleic Acids, 17, 185-197. https://doi.org/10.1016/j.omtn.2019.05.018

7. Jarroux, J., Morillon, A., \& Pinskaya, M. (2017). History, discovery, and classification of lncRNAs. Advances in Experimental Medicine and Biology, 1008, 1-46. https://doi.org/10.1007/ 978-981-10-5203-3_1

8. Zhang, Y., Du, W., \& Yang, B. (2019). Long non-coding RNAs as new regulators of cardiac electrophysiology and arrhythmias: Molecular mechanisms, therapeutic implications and challenges. Pharmacology \& Therapeutics, 203, 107389. https://doi.org/10. 1016/j.pharmthera.2019.06.011

9. Wang, M., Jiang, S., Yu, F., Zhou, L., \& Wang, K. (2019). Noncoding RNAs as Molecular Targets of Resveratrol Underlying Its Anticancer Effects. Journal of Agricultural and Food Chemistry, 67(17), 4709-4719. https://doi.org/10.1021/acs.jafc.9b01667

10. Tang, Y., \& Ji, F. (2019). lncRNA HOTTIP facilitates osteosarcoma cell migration, invasion and epithelial-mesenchymal transition by forming a positive feedback loop with c-Myc. Oncology Letters, 18(2), 1649-1656. https://doi.org/10.3892/ol.2019.10463

11. Rui, Y., Hu, M., Wang, P., Zhang, C., Xu, H., Li, Y., Zhang, Y., Gu, J., \& Wang, Q. (2019). LncRNA HOTTIP mediated DKK1 downregulation confers metastasis and invasion in colorectal cancer cells. Histology and Histopathology, 34(6), 619-630. https:// doi.org/10.14670/hh-18-043

12. Yuan, Q., Liu, Y., Fan, Y., Liu, Z., Wang, X., Jia, M., Geng, Z., Zhang, J., \& Lu, X. (2018). LncRNA HOTTIP promotes papillary thyroid carcinoma cell proliferation, invasion and migration by regulating miR-637. The International Journal of Biochemistry \& Cell Biology, 98, 1-9. https://doi.org/10.1016/j.biocel.2018.02. 013

13. Liao, B., Chen, R., Lin, F., Mai, A., Chen, J., Li, H., Xu, Z., \& Dong, S. (2018). Long noncoding RNA HOTTIP promotes endothelial cell proliferation and migration via activation of the Wnt/B-catenin pathway. Journal of Cellular Biochemistry, 119(3), 2797-2805. https://doi.org/10.1002/jcb.26448

14. Wang, Y., Li, G., Zhao, L., \& Lv, J. (2018). Long noncoding RNA HOTTIP alleviates oxygen-glucose deprivation-induced neuronal injury via modulating miR-143/hexokinase 2 pathway. Journal of Cellular Biochemistry, 119(12), 10107-10117. https://doi.org/10. $1002 /$ jcb. 27348

15. Fasolo, A., Sessa, C., Gianni, L., \& Broggini, M. (2013). Seminars in clinical pharmacology: an introduction to MET inhibitors for the medical oncologist. Annals of Oncology : Official Journal of the European Society for Medical Oncology, 24(1), 14-20. https:// doi.org/10.1093/annonc/mds520

16. Bahrami, A., Shahidsales, S., Khazaei, M., Ghayour-Mobarhan, M., Maftouh, M., Hassanian, S. M., \& Avan, A. (2017). C-Met as a potential target for the treatment of gastrointestinal cancer: Current status and future perspectives. Journal of Cellular Physiology, 232(10), 2657-2673. https://doi.org/10.1002/jcp.25794

17. Lee, C., Whang, Y. M., Campbell, P., Mulcrone, P. L., Elefteriou, F., Cho, S. W., \& Park, S. I. (2018). Dual targeting c-met and VEGFR2 in osteoblasts suppresses growth and osteolysis of prostate cancer bone metastasis. Cancer Letters, 414, 205-213. https:// doi.org/10.1016/j.canlet.2017.11.016

18. Chen, R. L., Zhao, J., Zhang, X. C., Lou, N. N., Chen, H. J., Yang, X., Su, J., Xie, Z., Zhou, Q., Tu, H. Y., Zhong, W. Z., Yan,
H. H., Guo, W. B., Wu, Y. L., \& Yang, J. J. (2018). Crizotinib in advanced non-small-cell lung cancer with concomitant ALK rearrangement and c-Met overexpression. BMC Cancer, 18(1), 1171. https://doi.org/10.1186/s12885-018-5078-y

19. Lee, S. J., Lee, J., Park, S. H., Park, J. O., Lim, H. Y., Kang, W. K., Park, Y. S., \& Kim, S. T. (2018). c-MET overexpression in colorectal cancer: A poor prognostic factor for survival. Clinical Colorectal Cancer, 17(3), 165-169. https://doi.org/10.1016/j.clcc. 2018.02.013

20. Liu, G., Hu, Y., Cheng, X., Wang, Y., Gu, Y., Liu, T., \& Shi, H. (2019). Volumetric parameters on (18)F-FDG PET/CT predict the survival of patients with gastric cancer associated with their expression status of c-MET. BMC Cancer, 19(1), 790. https://doi. org/10.1186/s12885-019-5935-3

21. Sato, T., Tani, Y., Murao, S., Fujieda, H., Sato, H., Matsumoto, M., Takeuchi, T., \& Ohtsuki, Y. (2001). Focal enhancement of expression of c-Met/hepatocyte growth factor receptor in the myocardium in human myocardial infarction. Cardiovascular Pathology: The Official Journal of the Society for Cardiovascular Pathology, 10(5), 235-240. https://doi.org/10.1016/s10548807(01)00079-5

22. Gallo, S., Sala, V., Gatti, S., \& Crepaldi, T. (2015). Cellular and molecular mechanisms of HGF/Met in the cardiovascular system. Clinical Science (London, England: 1979), 129(12), 1173-1193. https://doi.org/10.1042/cs20150502

23. Jiao, L., Li, M., Shao, Y., Zhang, Y., Gong, M., Yang, X., Wang, Y., Tan, Z., Sun, L., Xuan, L., Yu, Q., Li, Y., Gao, Y., Liu, H., Xu, H., Li, X., Zhang, Y., \& Zhang, Y. (2019). IncRNA-ZFAS1 induces mitochondria-mediated apoptosis by causing cytosolic $\mathrm{Ca}(2+)$ overload in myocardial infarction mice model. Cell Death \& Disease, 10(12), 942. https://doi.org/10.1038/ s41419-019-2136-6

24. Hu, H., Wu, J., Yu, X., Zhou, J., Yu, H., \& Ma, L. (2019). Long non-coding RNA MALAT1 enhances the apoptosis of cardiomyocytes through autophagy inhibition by regulating TSC2-mTOR signaling. Biological Research, 52(1), 58. https://doi.org/10.1186/ s40659-019-0265-0

25. Hu, H., Wu, J., Yu, X., Zhou, J., Yu, H., \& Ma, L. (2020). Long noncoding RNA MALAT1 enhances the apoptosis of cardiomyocytes through autophagy modulation. Biochemistry and Cell Biology, 98, 1-7. https://doi.org/10.1139/bcb-2019-0062

26. Zhang, Z., Li, H., Chen, S., Li, Y., Cui, Z., \& Ma, J. (2017). Knockdown of MicroRNA-122 Protects H9c2 Cardiomyocytes from Hypoxia-Induced Apoptosis and Promotes Autophagy. Medical Science Monitor : International Medical Journal of Experimental and Clinical Research, 23, 4284-4290. https://doi.org/10. 12659/msm.902936

27. He, Q., Fang, Y., Lu, F., Pan, J., Wang, L., Gong, W., Fei, F., Cui, J., Zhong, J., Hu, R., Liang, M., Fang, L., Wang, H., Yu, M., \& Zhang, Z.-F. (2019). Analysis of differential expression profile of miRNA in peripheral blood of patients with lung cancer. Journal of Clinical Laboratory Analysis. https://doi.org/10.1002/jcla. 23003

28. Liu, H., Li, G., Zhao, W., \& Hu, Y. (2016). Inhibition of MiR-92a May Protect Endothelial Cells After Acute Myocardial Infarction in Rats: Role of KLF2/4. Medical Science Monitor : International Medical Journal of Experimental and Clinical Research, 22, 2451-2462. https://doi.org/10.12659/msm.897266

29. Fan, Y. Z., Huang, H., Wang, S., Tan, G. J., \& Zhang, Q. Z. (2019). Effect of lncRNA MALAT1 on rats with myocardial infarction through regulating ERK/MAPK signaling pathway. European Review for Medical and Pharmacological Sciences, 23(20), 9041-9049. https://doi.org/10.26355/eurrev_201910_ 19306 
30. Uchida, S., \& Dimmeler, S. (2015). Long noncoding RNAs in cardiovascular diseases. Circulation Research, 116(4), 737-750. https://doi.org/10.1161/circresaha.116.302521

31. Yan, B., Yao, J., Liu, J. Y., Li, X. M., Wang, X. Q., Li, Y. J., Tao, Z. F., Song, Y. C., Chen, Q., \& Jiang, Q. (2015). IncRNA-MIAT regulates microvascular dysfunction by functioning as a competing endogenous RNA. Circulation Research, 116(7), 1143-1156. https://doi.org/10.1161/circresaha.116.305510

32. Chen, L., Yang, W., Guo, Y., Chen, W., Zheng, P., Zeng, J., \& Tong, W. (2017). Exosomal lncRNA GAS5 regulates the apoptosis of macrophages and vascular endothelial cells in atherosclerosis. PloS ONE, 12(9), e0185406. https://doi.org/10.1371/journ al.pone. 0185406

33. Liu, Z., Liu, J., Wei, Y., Xu, J., Wang, Z., Wang, P., Sun, H., Song, Z., \& Liu, Q. (2020). LncRNA MALAT1 prevents the protective effects of miR-125b-5p against acute myocardial infarction through positive regulation of NLRC5. Experimental and Therapeutic Medicine, 19(2), 990-998. https://doi.org/10.3892/ etm.2019.8309

34. Ong, S. B., Katwadi, K., Kwek, X. Y., Ismail, N. I., Chinda, K., Ong, S. G., \& Hausenloy, D. J. (2018). Non-coding RNAs as therapeutic targets for preventing myocardial ischemia-reperfusion injury. Expert Opinion on Therapeutic Targets, 22(3), 247-261. https://doi.org/10.1080/14728222.2018.1439015

35. Yang, J., Huang, X., Hu, F., Fu, X., Jiang, Z., \& Chen, K. (2019). LncRNA ANRIL knockdown relieves myocardial cell apoptosis in acute myocardial infarction by regulating IL-33/ST2. Cell cycle (Georgetown, Tex), 18(23), 3393-3403. https://doi.org/10.1080/ 15384101.2019.1678965

36. Li, X., Zhou, J., \& Huang, K. (2017). Inhibition of the lncRNA Mirt1 Attenuates Acute Myocardial Infarction by Suppressing NF- $\mathrm{kB}$ Activation. Cellular Physiology and Biochemistry : International Journal of Experimental Cellular Physiology, Biochemistry, and Pharmacology, 42(3), 1153-1164. https://doi.org/10. $1159 / 000478870$

37. Li, T., Tian, H., Li, J., Zuo, A., Chen, J., Xu, D., Guo, Y., \& Gao, H. (2019). Overexpression of lncRNA Gm2691 attenuates apoptosis and inflammatory response after myocardial infarction through PI3K/Akt signaling pathway. IUBMB Life, 71(10), 1561-1570. https://doi.org/10.1002/iub.2081

38. Su, Q., Liu, Y., Lv, X. W., Ye, Z. L., Sun, Y. H., Kong, B. H., \& Qin, Z. B. (2019). Inhibition of IncRNA TUG1 upregulates miR-142-3p to ameliorate myocardial injury during ischemia and reperfusion via targeting HMGB1- and Rac1-induced autophagy. Journal of Molecular and Cellular Cardiology, 133, 12-25. https://doi.org/10.1016/j.yjmcc.2019.05.021

39. Liao, B., Dong, S., Xu, Z., Gao, F., Zhang, S., \& Liang, R. (2020). LncRNA Kcnq1ot1 renders cardiomyocytes apoptosis in acute myocardial infarction model by up-regulating Tead1. Life Sciences, 256, 117811. https://doi.org/10.1016/j.lfs.2020.117811

40. Zhou, J., Li, D., Yang, B. P., \& Cui, W. J. (2020). LncRNA XIST inhibits hypoxia-induced cardiomyocyte apoptosis via mediating miR-150-5p/Bax in acute myocardial infarction. European Review for Medical and Pharmacological Sciences, 24(3), 13571366. https://doi.org/10.26355/eurrev_202002_20193
41. Yang, G., \& Lin, C. (2020). Long noncoding RNA SOX2-OT exacerbates hypoxia-induced cardiomyocytes injury by regulating miR-27a-3p/TGF $\beta$ R1 axis. Cardiovascular Therapeutics, 2020, 1-11. https://doi.org/10.1155/2020/2016259

42. Teng, Y., Ding, M., Wang, X., Li, H., Guo, Q., Yan, J., \& Gao, L. (2020). LncRNA RMRP accelerates hypoxia-induced injury by targeting miR-214-5p in H9c2 cells. Journal of Pharmacological Sciences, 142(2), 69-78. https://doi.org/10.1016/j.jphs.2019.07. 014

43. Liang, H., Li, F., Li, H., Wang, R., \& Du, M. (2021). Overexpression of lncRNA HULC attenuates myocardial ischemia/reperfusion injury in rat models and apoptosis of hypoxia/reoxygenation cardiomyocytes via targeting miR-377-5p through NLRP3/Caspase-1/IL-1 $\beta$ signaling pathway inhibition. Immunological Investigations, 50(8), 925-938. https://doi.org/10.1080/08820139.2020. 1791178

44. Zhang, J., Gao, C., Meng, M., \& Tang, H. (2016). Long noncoding RNA MHRT protects cardiomyocytes against $\mathrm{H} 2 \mathrm{O} 2$-induced apoptosis. Biomolecules \& Therapeutics, 24(1), 19-24. https:// doi.org/10.4062/biomolther.2015.066

45. Han, F., Chen, Q., Su, J., Zheng, A., Chen, K., Sun, S., Wu, H., Jiang, L., Xu, X., Yang, M., Yang, F., Zhu, J., \& Zhang, L. (2019). MicroRNA-124 regulates cardiomyocyte apoptosis and myocardial infarction through targeting Dhcr24. Journal of Molecular and Cellular Cardiology, 132, 178-188. https://doi.org/10.1016/j. yjmcc.2019.05.007

46. Guo, J., Liu, H. B., Sun, C., Yan, X. Q., Hu, J., Yu, J., Yuan, Y., \& Du, Z. M. (2019). MicroRNA-155 Promotes Myocardial Infarction-Induced Apoptosis by Targeting RNA-Binding Protein QKI. Oxidative Medicine and Cellular Longevity, 2019, 4579806. https://doi.org/10.1155/2019/4579806

47. Yin, Y., Lv, L., \& Wang, W. (2019). Expression of miRNA-214 in the sera of elderly patients with acute myocardial infarction and its effect on cardiomyocyte apoptosis. Experimental and Therapeutic Medicine, 17(6), 4657-4662. https://doi.org/10.3892/etm.2019. 7464

48. Cheng, J., Wu, L. M., Deng, X. S., Wu, J., Lv, Z., Zhao, H. F., Yang, Z., \& Ni, Y. (2018). MicroRNA-449a suppresses hepatocellular carcinoma cell growth via G1 phase arrest and the HGF/ MET c-Met pathway. Hepatobiliary \& Pancreatic Diseases International : HBPD INT, 17(4), 336-344. https://doi.org/10.1016/j. hbpd.2018.07.006

49. Xu, X., Zhu, Y., Liang, Z., Li, S., Xu, X., Wang, X., Wu, J., Hu, Z., Meng, S., Liu, B., Qin, J., Xie, L., \& Zheng, X. (2016). c-Met and CREB1 are involved in miR-433-mediated inhibition of the epithelial-mesenchymal transition in bladder cancer by regulating Akt/GSK-3ß/Snail signaling. Cell Death \& Disease, 7(2), e2088. https://doi.org/10.1038/cddis.2015.274

50. Hoetelmans, R., van Slooten, H. J., Keijzer, R., Erkeland, S., van de Velde, C. J., \& Dierendonck, J. H. (2000). Bcl-2 and Bax proteins are present in interphase nuclei of mammalian cells. Cell Death and Differentiation, 7(4), 384-392. https://doi.org/10.1038/ sj.cdd. 4400664

Publisher's Note Springer Nature remains neutral with regard to jurisdictional claims in published maps and institutional affiliations. 